\title{
Modeling Chemotherapy-Induced Peripheral Neuropathy Using a Nerve-on-a-Chip Microphysiological System
}

\author{
Liana Kramer', Hieu T. Nguyen', Elizabeth Jacobs ${ }^{1}$, Laurie McCoy ${ }^{1}$, J. Lowry Curley', Anup D. Sharma ${ }^{1}$ \\ and Michael J. Moore 1,2,3 \\ ${ }^{1}$ AxoSim Inc., New Orleans, LA, USA; ${ }^{2}$ Dept. of Biomedical Engineering, Tulane University, New Orleans, LA, USA; ${ }^{3}$ Brain Institute, Tulane \\ University, New Orleans, LA, USA
}

\begin{abstract}
Organ-on-a-chip devices that mimic in vivo physiology have the potential to identify effects of chemical and drug exposure in early preclinical stages of drug development while relying less heavily on animal models. We designed a hydrogel rat nerve-on-a-chip ( $\mathrm{RNoaC}$ ) construct that promotes axon growth analogous to mature nerve anatomy and is the first 3D in vitro model to collect electrophysiological and histomorphic metrics to assess in vivo pathophysiology. Here we culture embryonic rat dorsal root ganglia (DRG) in the construct to demonstrate its potential to screen for implications of nerve dysfunction in chemotherapy-induced peripheral neuropathy (CIPN). RNoaC constructs containing DRG explants from E 15 rat pups were exposed to the chemotherapeutics bortezomib, oxaliplatin, paclitaxel or vincristine for 7 days. Then, axons were electrically stimulated to collect nerve conduction velocity (NCV) and peak amplitude (AMP), which are clinical electrophysiological metrics indicative of healthy or diseased populations. All chemotherapeutics decreased NCV and AMP in a concentration-dependent manner. At high drug concentrations, NCV and AMP were 10-60\% lower than control values. Histopathological analysis revealed hallmarks of peripheral neuropathy. $I \mathrm{C}_{50}$ values calculated from concentration-response curves indicate that the significant decrease in function occurred before a decrease in viability. Our data suggest that electrophysiology recordings collected from our RNoaC platform can closely track subtle pathological changes in nerve function. The ability to collect clinically relevant data from $\mathrm{RNoaC}$ suggests it can be an effective tool for in vitro preclinical screening for peripheral neuropathy.
\end{abstract}

\section{Introduction}

Experimental drugs have unacceptably high attrition rates during development, from discovery to clinical trials and then to market, with only $9.6 \%$ of drugs successfully reaching patients (Thomas, 2016). Once accepted to market, one-in-three drugs receives US Food and Drug Administration (FDA) mandated strict warning black box labels or is withdrawn completely for safety reasons within 25 years of approval (Frank et al., 2014). Toxicity is the second leading reason that drugs are removed from market with neurotoxicity responsible for $16 \%$ of all withdrawals (Onakpoya et al., 2016).

Peripheral nerves are particularly susceptible to toxic off-target effects that manifest as sensory and motor deficits and can result in permanent nerve damage (Manji, 2013). A common toxic off-target effect is chemotherapy-induced peripheral neuropathy
(CIPN), which is rapidly induced after the administration of anti-cancer drugs (Argyriou et al., 2012; Cavaletti and Marmiroli, 2010). Patients with CIPN may experience a range of sensory symptoms including spontaneous tingling, burning pain, and joint and muscle pain in the distal extremities in a "glove and stocking" distribution (Peters et al., 2007). Often, these side effects discourage patient use of the drug, leading to delays or limited dosages and even discontinued treatment altogether (Staff et al., 2017). In some patients, these symptoms resolve following discontinuation of therapy, but an estimated $30 \%$ of patients are left with permanent symptoms that affect their overall quality of life (Rivera and Cianfrocca, 2015). Drugs with neurotoxic side effects reaching market approval is attributed to a lack of reliable screens for drug candidate neurotoxicity.

Traditional methods for studying neuronal damage and screening neurotoxicity have largely relied on cell and animal models 
A

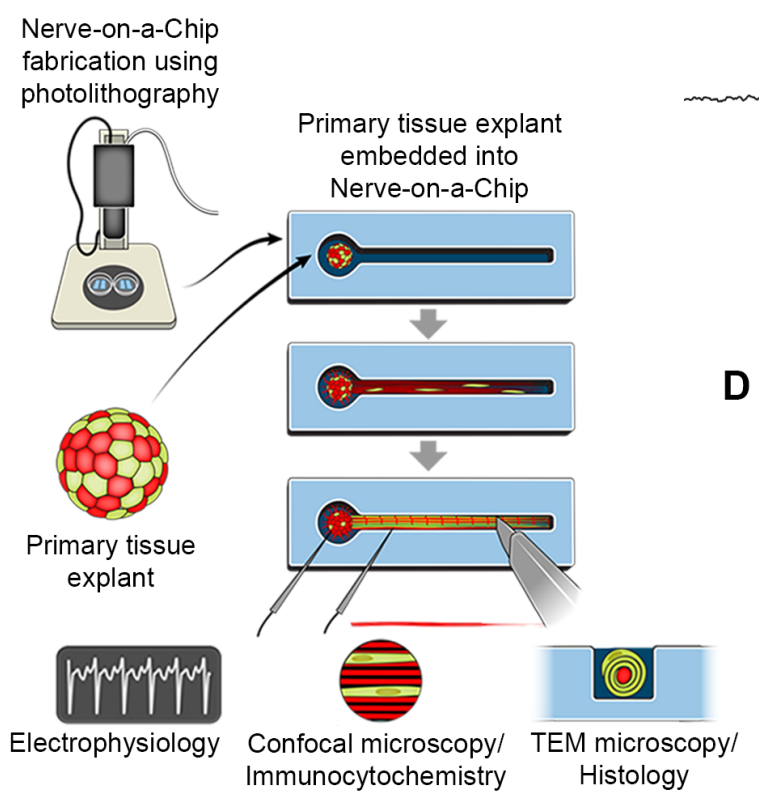

C

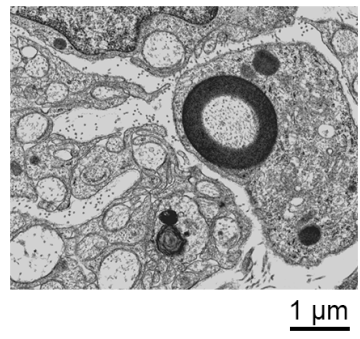

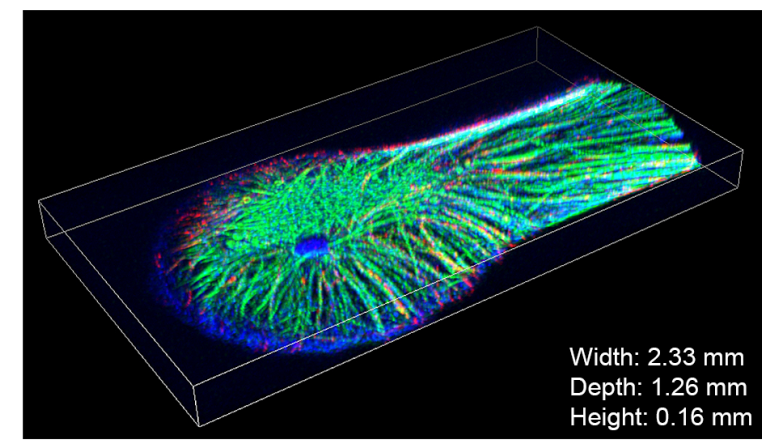

Fig. 1: Study design

A) Study design showing the process of fabricating a nerve-on-a-chip construct in which the dual hydrogel is polymerized using photolithography and how the DRG explant is embedded and matures. The various means by which the system may be characterized include B) electrophysiology, C) TEM of histological tissue sections (scale bar: $1 \mu \mathrm{m}$ ), and D) confocal microscopy. Graphic credits (A) to Anita Impagliazzo. All rights reserved, used with permission.

(Brewer et al., 2016). In vitro cell models of peripheral nervous system dysfunction and injury classically use primary dorsal root ganglia (DRG) to study the mechanisms underlying drug-induced neurotoxicity based on analyzing cell viability, morphology and evoked electrical activity (Fukuda and Segal, 2017). However, these models do not provide an accurate representation of the complexities of in vivo neurotoxicity, since in vitro models often poorly mimic natural tissue organization and can be difficult to analyze by histopathology (Kapałczyńska et al., 2018; Golden and Johnson, 2004).

Many of the limitations of traditional cell culture can be addressed by using animal models in preclinical studies. Animals have biochemical and biomechanical microenvironments that are more similar to those of their human counterparts and can be used to evaluate peripheral neuropathy risk with a variety of outcome measures that cannot be measured in vitro. Although animal studies demonstrate many neuropathological changes that are similar to human disease, including electrophysiological dysfunction and behavioral changes consistent with neuropathic pain, significant differences in these abnormalities are observed across laboratories (Brewer et al., 2016; Höke and Ray, 2014; Golden and Johnson, 2004). These inconsistencies can be attributed to the lack of standardization for animal strain, age, weight, sex, or drug dosing schedule (Fukuda and Segal, 2017). These discrepancies in studies call into question whether live animals are the most faithful model for studying human peripheral sensory nerve disease (Li et al., 2013; Höke, 2012; Jones et al., 2018).

The key to developing novel therapies to overcome neuropathy will be the use of relevant tissue models that recapitulate key neuropathic features and can reliably predict clinically relevant metrics of toxicity (Brewer et al., 2016). Thus, 3D organon-chip models have rapidly gained traction for developing predictive cell-based assays that are suitable for drug development and toxicity screening. These models have a controllable microenvironment, mimic microphysiology, and can be evaluated for drug efficacy and toxicity, which are nearly impossible to measure with traditional drug screening methods (Ghaemmaghami et al., 2012; Kimlin et al., 2013). Additionally, these models can be used to collect metrics in a faster and more inexpensive manner compared to labor-intensive and expensive in vivo studies.

We have developed novel, cell-based 3D in vitro rat and human nerve-on-a-chip models of the peripheral nervous system for preclinical neurotoxicity testing and disease modeling, specifically as medium-throughput, high-content screens. Our previously described nerve-on-a-chip constructs exhibit structural and functional characteristics that closely mimic peripheral nerve 
fibers found in vivo (Huval et al., 2015; Khoshakhlagh et al., 2018; Sharma et al., 2019). Most unique to the nerve-on-a-chip construct is the ability to collect relevant metrics that correlate directly with clinical in vivo pathophysiology, such as nerve conduction velocity and histomorphometry.

Here, we culture rat dorsal root ganglia (DRG) in the rat nerveon-a-chip (RNoaC) to demonstrate its potential as a preclinical assay for screening for drug-induced nerve dysfunction by measuring electrical signals in tissue exposed to four chemotherapeutic agents known to cause peripheral neuropathy. We additionally show that the RNoaC tissues exhibit histopathological hallmarks of neuropathy and that the platform is compatible with common in vitro techniques (Fig. 1). The $\mathrm{RNoaC}$ is an inexpensive system for preclinical testing in comparison to in vivo rat studies and can be used for longer chronic treatment regimens than traditional $2 \mathrm{D}$ in vitro models.

\section{Materials and methods}

\section{Fabrication of $3 D$ dual hydrogel system}

The dual hydrogel culture system was fabricated on the membranes of Transwell ${ }^{\circledR}$ 6-well inserts $(0.4 \mu \mathrm{m} / \mathrm{PES}$; Corning) using digital projection photolithography as previously described (Curley and Moore, 2011; Bowser and Moore, 2019). The outer cell-impermeable hydrogel was created using a photo-translinkable solution of $10 \% \mathrm{w} / \mathrm{v}$ polyethylene glycol dimethacrylate 1000 (PEGDMA; Polysciences, Warrington, PA) and $0.55 \mathrm{mM}$ lithium phenyl-2,4,6-trimethylbenzoylphosphinate (LAP; SigmaAldrich, St. Louis, MO). The photo-translinkable solution was irradiated for 25-30 sec using ultraviolet light of $385 \mathrm{~nm}$ wavelength at $300 \mathrm{~W} / \mathrm{m}^{2}$ with a digital micromirror device (DMD) light engine (PRO4500 Wintech Digital Systems Technology Corp, Carlsbad, CA). A mask design and polymerization parameters were selected using commercial software (DLP Lightcrafter 4500 Control Software, Texas Instruments, Dallas, TX). The mask shape resembles a keyhole, where the circular reservoir is referred to as the "bulb" and the narrow slot is the "channel". The constructs were washed using $2 \%$ antibiotic/antimycotic wash buffer (Thermo Fischer Scientific, Walton, MA) and the channels were filled with $8 \%$ Growth Factor-Reduced Matrigel ${ }^{\circledR}$ Matrix (Corning, Corning, NY) to create a cell-permeable scaffold.

\section{Primary tissue culture in the dual hydrogel system}

All procedures involving animals adhered to guidelines set forth by the American Veterinary Medical Association and were approved by the Institutional Animal Care and Use Committee (IACUC) of Tulane University. Timed-pregnant adult female Long Evans rats approximately 8 weeks of age were purchased from Charles River Laboratories and housed by the Tulane University Department of Comparative Medicine, where they were kept for no more than 2 days in single cages and given food and water ad libitum. At gestation day E15, animals were deeply anesthetized with a sterile IP injection of a mixture of ketamine $(80 \mathrm{mg} / \mathrm{kg})$ and xylazine $(8 \mathrm{mg} / \mathrm{kg})$. A cesarean section was performed, the uterus and embryos were removed together, and then the animal was euthanized by an overdose of potassium chloride solution $(100 \mathrm{mg} / \mathrm{kg})$ administered by intracardiac injection. Rat embryos were euthanized by rapid decapitation, and the DRGs were immediately harvested by removing the spinal column and detaching the DRGs. Approximately 30 DRGs were removed per embryo, and individual DRG explants were cultured in the hydrogel constructs. DRGs were placed into the center of the keyhole-shaped channel. Constructs were immersed in neurobasal medium supplemented with B27 (2\% v/v), L-glutamine (1\% v/v), recombinant rat $\beta$-subunit nerve growth factor $(2.5 \mathrm{~S} \mathrm{NGF})(10 \mathrm{ng} /$ $\mathrm{ml})$, and antibiotic/antimycotic solution (A/A) (1\% v/v) containing penicillin, streptomycin, and amphotericin (all from Life Technologies, CA, USA) overnight to allow the tissue to adhere to the insert. The following day, cultures were changed to pre-myelination media in which they were cultured for 7 days to promote Schwann cell migration and neurite outgrowth (Basal Medium Eagle (BME) supplemented with insulin-transferrin-selenium (ITS) $(1 \% \mathrm{v} / \mathrm{v})$, L-glutamine ( $1 \% \mathrm{v} / \mathrm{v})$, D-glucose $(4 \mathrm{~g} / \mathrm{L})$, bovine serum albumin $(0.2 \% \mathrm{w} / \mathrm{v}), \mathrm{NGF}(10 \mathrm{ng} / \mathrm{ml})$ and $\mathrm{A} / \mathrm{A}(1 \% \mathrm{v} / \mathrm{v}))$. Following the pre-myelination period, the medium was changed to a supplement-rich myelination medium composed of BME supplemented with ITS $(1 \% \mathrm{v} / \mathrm{v})$, L-glutamine $(1 \% \mathrm{v} / \mathrm{v})$, fetal bovine serum $(15 \% \mathrm{v} / \mathrm{v})$, D-glucose (4 g/L), NGF (10 ng/ml), L-ascorbic acid $(50 \mu \mathrm{g} / \mathrm{ml})$ and $\mathrm{A} / \mathrm{A}(1 \% \mathrm{v} / \mathrm{v})$ to promote tissue growth and axon extension for an additional 21 days.

After a total of 4 weeks in culture, tissue constructs had achieved robust axon growth and distinctive myelination, at which point they were challenged with the chemotherapeutics paclitaxel (Ptx), bortezomib (Bz), oxaliplatin $(\mathrm{Ox})$ or vincristine $(\mathrm{Vn})$ or with non-neuropathic acetaminophen and amoxicillin. Vn was dissolved in water, all other drugs were dissolved in DMSO (all from Sigma-Aldrich). The concentration ranges were selected from concentration-response curves found in the literature and further narrowed based on small scale pilot experiments (data not shown). Experimentally determined $\mathrm{IC}_{50}$ values of chemotherapeutics for electrophysiology and cell viability were then used in the following experiments. Constructs were exposed to drugs for 7 days in supplement-free medium (BME, D-glucose $(4 \mathrm{~g} / \mathrm{L})$, and A/A $(1 \% \mathrm{v} / \mathrm{v}))$ with a half medium change and redose mid-week. Supplements were removed for the treatment period to avoid possible neuroprotective effects of these nutrients and growth factors.

\section{Electrophysiology}

$\mathrm{RNoaC}$ constructs were submerged in oxygenated artificial cerebrospinal fluid (ACSF; $170 \mathrm{mM} \mathrm{NaCl}, 7 \mathrm{mM} \mathrm{KCl}, 37 \mathrm{mM}$ $\mathrm{NaHCO}_{3}, 0.91 \mathrm{mM} \mathrm{Na} \mathrm{HPO}_{4} \cdot 7 \mathrm{H}_{2} \mathrm{O}, 14 \mathrm{mM} \mathrm{D}(+)$-glucose, $4 \mathrm{mM} \mathrm{MgSO}_{4}$, and $2 \mathrm{mM} \mathrm{CaCl}_{2}$ in deionized water) for electrophysiological recording at room temperature (RT). Axons growing in the channel were stimulated with a concentric bipolar platinum-iridium electrode positioned 1-5 mm distal to the bulb of the channel, and a pulled glass micropipette electrode (1-4 M $\Omega$ ) was inserted into the bulb near the clustered cell bodies to record compound action potentials (CAPs) (Fig. 2). Unlike in clinical measurements, we place the recording electrode at the DRG body rather than along the axons to prevent damag- 


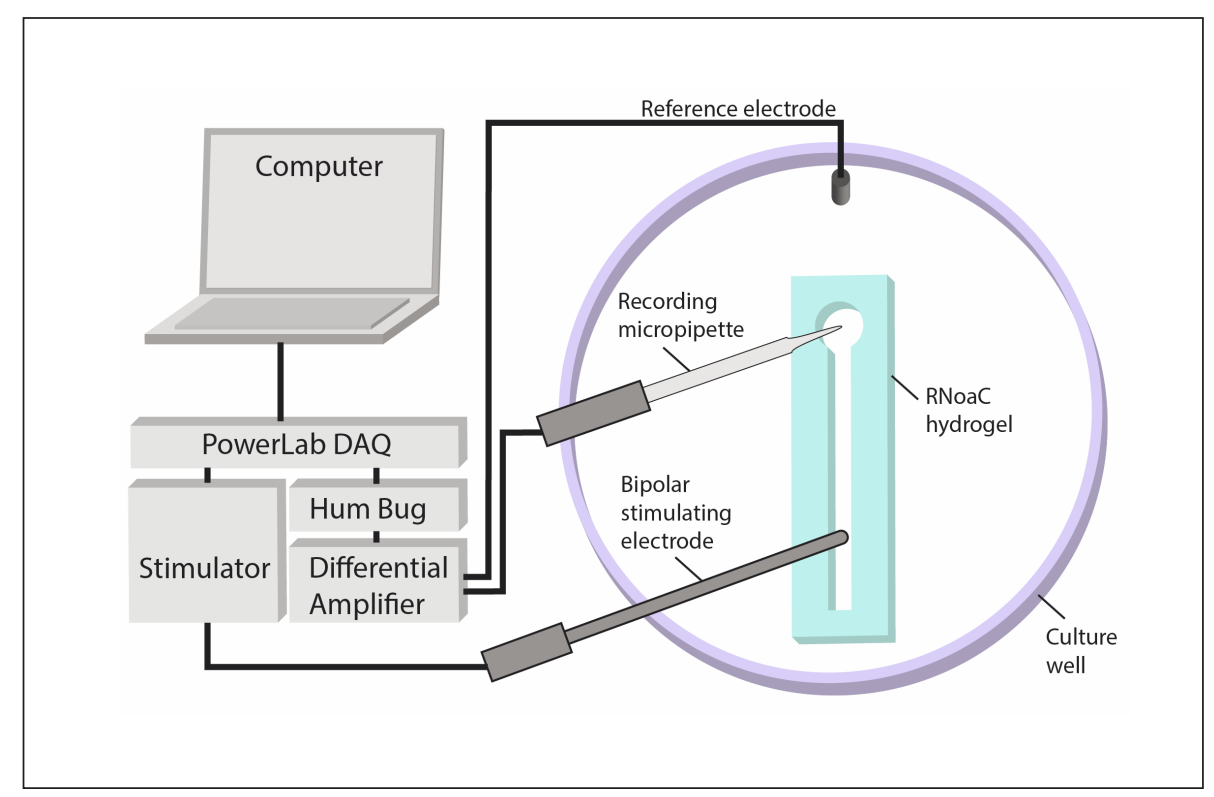

Fig. 2: Schematic of electrophysiology set up

Pre-set electrical parameters on the computer are converted to an analog signal by the PowerLab $\mathrm{DAQ}$, and the stimulator delivers a $1 \mathrm{~Hz}$ electrical pulse through the bipolar stimulating electrode. The action potentials are recorded with the micropipette, amplified and de-noised with the differential amplifier and Hum Bug, respectively, converted to a digital signal by the PowerLab DAQ, and visualized with the computer's LabChart software.

ing the axon structures and because the somata generate stronger signal amplitudes that are easily recordable. The reference electrode was a $1 \mathrm{~mm}$ x $2.5 \mathrm{~mm} \mathrm{AgCl}$ pellet (A-M Systems, Catalog \#550010) submerged in ACSF at the edge of the well. An $\mathrm{AgCl}$ metal wire was placed in the ACSF-filled glass micropipette and was connected to a Model 3000 AC/DC Differential Amplifier (A-M Systems, Sequim, WA) set at 50x gain and $0.1 \mathrm{~Hz}$ high pass to $3 \mathrm{kHz}$ low pass filtering. Electrical interference was removed with the Hum Bug Noise Eliminator (Quest Scientific, North Vancouver, Canada). Monophasic stimulation pulse height and width were kept at $-10 \mathrm{~V}$ and $200 \mu \mathrm{sec}$, respectively. Samples were stimulated at a rate of $1 \mathrm{~Hz}$, and at least 50 stimulations were applied per sample. Using an analog-to-digital converter (PowerLab; AD Instruments, Colorado Springs, CO), CAP waveforms were visualized and further analyzed using LabChart software (AD instruments). Analog CAP recordings were immediately digitized, and the digital signals were averaged. A stereomicroscope and camera were used to measure the distance between the stimulating and recording electrodes to calculate the nerve conduction velocity (NCV). Latency was measured as the time between the stimulus artifact and the first CAP peak having at least twice the amplitude of the noise. NCV was evaluated by dividing the distance between stimulating and recording electrodes by latency.

\section{Cell viability assays}

The Pierce ${ }^{\mathrm{TM}}$ LDH Cytotoxicity Assay (Thermo Fischer Scientific) was used according to the manufacturer's instructions to measure lactate dehydrogenase released from damaged cells into the medium. In brief, medium samples from the constructs were taken on day 2, 4 and 7 of drug exposure and incubated with the appropriate kit reagents for $30 \mathrm{~min}$ in the dark at RT. The absorbance was then measured with a Multiskan GO Microplate Spectrophotometer (Thermo Fischer Scientific) at $490 \mathrm{~nm}$.
The Cell Counting Kit-8 (CCK-8) was used to measure the metabolic activity of living cells. On day 7, DRGs were biopsied from the construct using a $1 \mathrm{~mm}$ biopsy punch (Miltex, Plainsboro, NJ) and the CCK-8 assay (Dojindo Laboratories, Kumamoto, Japan) was performed on the biopsies according to the manufacturer's instructions. In brief, the DRG biopsies were incubated with the appropriate kit reagents for $3 \mathrm{~h}$, and the absorbance was then measured with a Multiskan GO Microplate Spectrophotometer (Thermo Fischer Scientific) at $450 \mathrm{~nm}$.

\section{Immunocytochemistry}

RNoaC samples treated with chemotherapeutics at their NCV $\mathrm{IC}_{50}$ concentrations were fixed with $4 \%$ paraformaldehyde (PFA; Electron Microscopy Sciences, Hatfield, PA). After fixation, samples were permeabilized in graded methanol/PBS washes on ice (Sigma-Aldrich). After a 5-min wash in 1\% Triton-X-100, samples were then placed in a blocking solution containing PBS, $5 \%$ normal goat serum (Jackson ImmunoResearch, West Grove, PA), $0.2 \%$ Triton-X-100 (Sigma-Aldrich), and $0.4 \%$ bovine serum albumin (Sigma-Aldrich) for $1 \mathrm{~h}$ at RT, followed by labeling with the following primary antibodies overnight in blocking solution at $4{ }^{\circ} \mathrm{C}$ : polyclonal rabbit- $\alpha-\beta$ III tubulin $(1: 300$; Abcam, Cambridge, MA) and monoclonal mouse- $\alpha$-myelin basic protein (MBP, 1:300; Biolegend, San Diego, CA). The following day, samples were labeled with secondary antibodies, polyclonal Alexa 488 goat anti-rabbit IgG (1:500, Invitrogen, Carlsbad, CA) or polyclonal Alexa 568 goat anti-mouse IgG (1:500, Abcam), and DAPI (1:300, Sigma-Aldrich). Microscopy was performed using a Nikon A1 confocal microscope (Nikon, Tokyo, Japan).

\section{Mitochondrial membrane potential assay}

The TMRE Mitochondrial Membrane Potential Assay (G-Biosciences, St. Louis, MO) was used to quantify changes in mitochondrial membrane potential of live cells. RNoaC constructs were 
exposed to either chemotherapeutic drugs at $\mathrm{NCV} \mathrm{IC}_{50}$ concentrations for 7 days or $20 \mu \mathrm{M}$ of carbonyl cyanide 4-(trifluoromethoxy) phenylhydrazone (FCCP) for $20 \mathrm{~min}$. FCCP induces depolarization in mitochondria and thus served as a positive control. $50 \mathrm{nM}$ TMRE dye in basal medium was added, and RNoaC constructs were incubated for $24 \mathrm{~h}$ on a shaker plate. The following day, the TMRE dye was replaced with 1xMMP Assay Buffer and the samples were imaged on a Nikon eclipse Ts2 epifluorescent inverted microscope (Nikon, Tokyo, Japan). Four random fields of view were taken per RNoaC. Fluorescent signal intensity was determined using a custom Image J (NIH, Bethesda, MD) macro to calculate the mean pixel intensity of thresholded images.

\section{Plastic resin embedding}

$\mathrm{RNoaC}$ samples treated with the chemotherapeutics at their respective $\mathrm{NCV} \mathrm{IC}_{50}$ concentrations were embedded in plastic resin for sectioning and imaging. All materials used for embedding were purchased from Electron Microscopy Sciences. Samples were fixed with $4 \%$ PFA $/ 0.5 \%$ glutaraldehyde for $30 \mathrm{~min}$ at RT. Secondary fixation and staining of cellular lipids was achieved by post-fixation with $1 \%$ osmium tetroxide in PBS for $2 \mathrm{~h}$ in the dark. The constructs were then washed with PBS prior to counterstaining with $2 \%$ aqueous uranyl acetate for $30 \mathrm{~min}$ in the dark. Dehydration was done with graded ethanol washes at RT. The following day, the constructs were washed twice with 100\% ethanol for $30 \mathrm{~min}$.

Hydrogel constructs were dissected individually from the transmembrane wells and placed in Flat Embedding Molds (EMS 70902, Electron Microscopy Sciences). Remaining ethanol was given time to evaporate from the fixed hydrogels before replacement with infiltration medium consisting of a 1:1 mixture of Spurr's resin (Low Viscosity Embedding Media Spurr's Kit; Electron Microscopy Sciences) and propylene oxide. Infiltration medium was left for $45 \mathrm{~min}$ before it was replaced by $100 \%$ Spurr's Resin, which was cured overnight in a $70^{\circ} \mathrm{C}$ oven and then for $48 \mathrm{~h}$ at RT before ultramicrotome sectioning.

\section{Sectioning, light microscopy (LM) and transmission electron microscopy (TEM)}

Ultrathin sections were cut using a Leica EM U27 microtome (Leica, Wetzlar, Germany) at four locations within the specimen: two locations within the bulb region, where the bulb meets the channel, and the proximal channel. These locations are approximately $1 \mathrm{~mm}$ apart along the construct. For LM, $0.5 \mu \mathrm{m}$ sections prepared by staining with $0.5 \%$ toluidine blue in $2 \%$ sodium borate were imaged using a Leica DM6 B microscope (Leica, Wetzlar, Germany). For TEM, 80-100 nm sections were placed on Formvar carbon-coated copper grids, 200 mesh, and impregnated with metal by floating on droplets of $2 \%$ uranyl acetate for 20 min at RT. They were then rinsed with deionized water droplets 3 times for 1 min. A JEOL 1400 TEM (Peabody, MA) with an accelerating voltage of $120 \mathrm{kV}$ was used to visualize samples at varying magnifications.

\section{Histomorphometric analysis}

TEM micrographs were analyzed using Nikon NIS-Elements BR Analysis software. Measurement of G-ratio, myelinated fiber di- ameter, and nonmyelinated fiber diameter were calculated using binary thresholding of the cross-sectional images. G-ratio is defined as the ratio of the inner to the outer diameter of the myelin sheath. The researcher performing histomorphometry was blinded to the samples' conditions throughout the data collection process.

To gather G-ratio and myelinated fiber diameter, a mask of the darkened myelin sheath was created using thresholding. Dark axonal mitochondria, axonal fibers, or Schwann cell organelles included in the thresholding were removed from the mask. Sheaths that lacked axons or contained severely degenerated axons were excluded from the above analyses. The number of sheaths excluded for this reason was recorded to calculate "\% myelin sheaths with degenerated axons." Additionally, the number of abnormal sheaths was recorded to arrive at " $\%$ abnormal myelin." Features such as myelin balloons twice the size of the inner axon, excessive cytoplasm in the lamellae or redundant myelin spanning at least three times the diameter of the inner axon were taken to be abnormal features. The numbers of abnormal and healthy sheaths were recorded over the area of each analyzed micrograph to yield myelinated fiber density.

To measure nonmyelinated fiber diameter and density, two fields from each sectioning level depicting at least 5 clearly distinguishable axons were selected at random ( 8 images per sample, 2 samples per condition). Thresholding separated nonmyelinated axons from darker surrounding Remak Schwann cells or extracellular matrix. The area of the binary objects was recorded, and nonmyelinated axon diameter was calculated. The number of axons measured was recorded over the area of each analyzed micrograph to give nonmyelinated fiber density.

Slides stained with toluidine blue were used to identify eccentric nuclei. Three sections from within the bulb region and three sections from where the bulb meets the channel were analyzed. Only nuclei that contained a visible nucleolus in the section were scored, and nuclei with severe chromatin condensation as a result of cell death were excluded from analysis but were noted. A nucleus was recorded as eccentric when the nucleolus appeared to touch the nuclear envelope or where the nucleolus fell into the outer half of the nucleus.

\section{Statistics}

One-way analysis of variance (ANOVA) with Dunnett's posthoc test was conducted using GraphPad Prism software (GraphPad Software, Inc., La Jolla, CA). A p-value $\leq 0.05$ was considered significant.

\section{Results}

\subsection{Electrophysiological activity of evoked nerve constructs is sensitive to drug exposure}

Compound action potentials (CAPs) were measured from RNo$\mathrm{aC}$ constructs by electrically stimulating axons distally while recording extracellular field potentials at the ganglion (within the bulb region). From the CAPs, nerve conduction velocity (NCV) and the peak amplitude (AMP), two electrophysiological clinical metrics indicative of healthy or diseased populations, were col- 
A

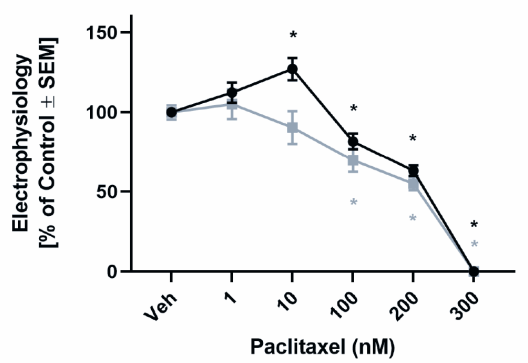

C

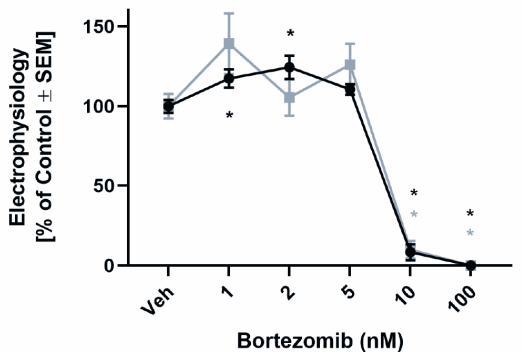

E

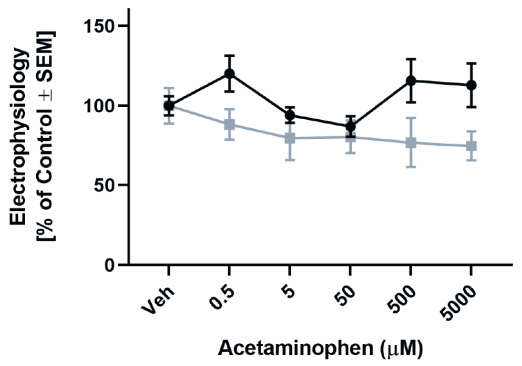

B

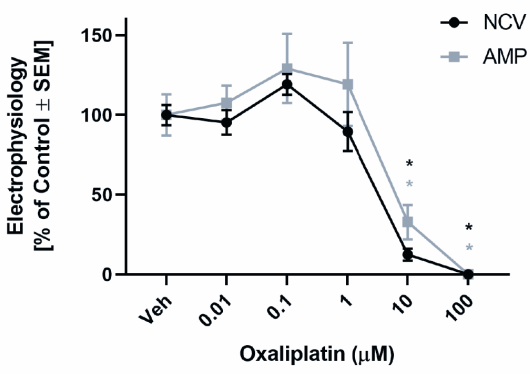

D

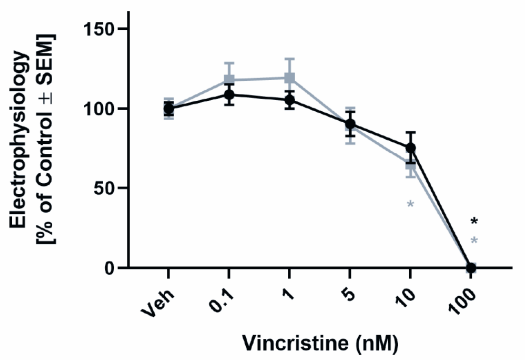

$\mathbf{F}$

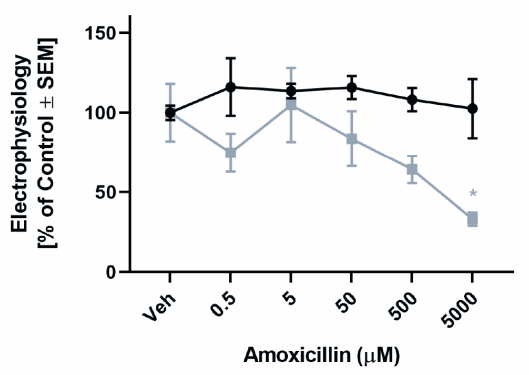

Fig. 3: Concentration response curves of evoked nerve constructs demonstrate decreases in clinically relevant electrophysiological metrics Nerve conduction velocity (NCV, black circles) and amplitude (AMP, gray squares) were calculated from waveforms of recorded compound action potentials (CAPs) of nerve constructs exposed to chemotherapeutics with known neurotoxic effects (A-D) or compounds not known to induce neuropathy (E-F). $\mathrm{N} \geq 8$; error bars represent SEM. *, $p$-value $\leq 0.05$ by one-way ANOVA followed by Dunnett's post-hoc test. Statistical comparisons made to vehicle control. lected. The first positive or negative spike recorded after stimulation with an amplitude of at least twice the noise level (signal to noise ratio $>2$ ) was considered to be the first peak and was used to calculate the NCV and AMP.

Mature RNoaC constructs were exposed to chemotherapeutics for 7 days with a half medium change and re-dose midweek. NCV and AMP decreased in a concentration-dependent manner across all chemotherapeutic drugs. At low drug concentrations, NCV and AMP measurements were typically similar to vehicle controls - approximately $0.30 \pm 0.02 \mathrm{~m} / \mathrm{s}$ and $105 \pm 14 \mu \mathrm{V}$, respectively. No significant decreases in electrical function were seen at concentrations lower than $10 \mathrm{nM}(\mathrm{Ptx}), 0.1 \mu \mathrm{M}(\mathrm{Ox})$, and $5 \mathrm{nM}(\mathrm{Bz}$ and $\mathrm{Vn})$. At higher drug concentrations, NCV and AMP dropped lower than the vehicle controls until reaching a complete loss of electrical activity at $300 \mathrm{nM}(\mathrm{Ptx}), 100 \mu \mathrm{M}$ (Ox), or $100 \mathrm{nM}$ (Bz and Vn) (Fig. 3). Low concentrations of paclitaxel and bortezomib produced an initial increase in NCV before values dropped at the higher concentrations (Fig. 3A,C).
Acetaminophen and amoxicillin, which are not known to have neuropathic effects, were employed as negative controls. Both compounds had minor effects on NCV and AMP, with only amoxicillin at the highest concentration of $5000 \mu \mathrm{M}$ causing a statistically significant decrease in AMP (Fig. 3E,F). All raw values of calculated NCV and AMP are presented in Tab. $\mathrm{S}^{1}$.

\subsection{Cell viability is maintained while electrophysiological metrics begin to decrease}

In order to ensure that decreases in electrophysiological measurements were due to changes in electrical function rather than a result of cell death, two cell viability assays were performed on the constructs exposed to the chemotherapeutic compounds.

The CCK-8 assay revealed a concentration-dependent decrease in cellular metabolic activity after constructs had been exposed to chemotherapeutics for 7 days. This effect was pronounced for $\mathrm{Ox}$ and $\mathrm{Bz}$, with $100 \mu \mathrm{M}$ Ox decreasing metabolic activity to $31 \%$ of the control and $1,000 \mathrm{nM} \mathrm{Bz}$ decreasing activity to $37 \%$

\footnotetext{
1 doi:10.14573/altex.2001181s
} 


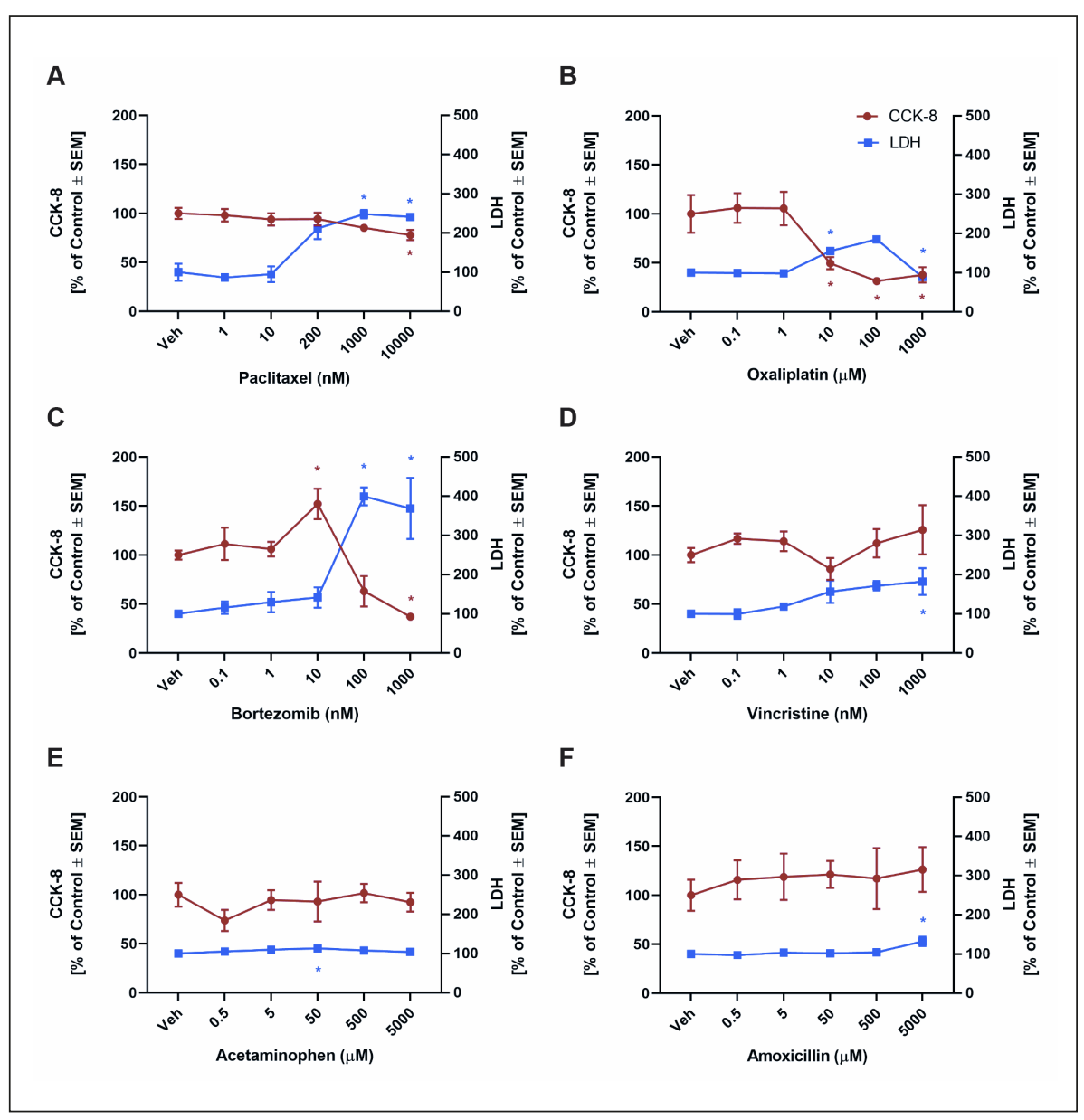

A

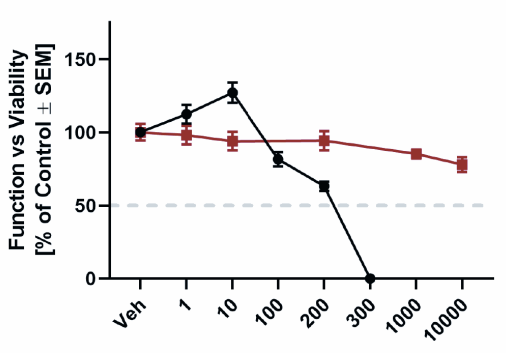

C

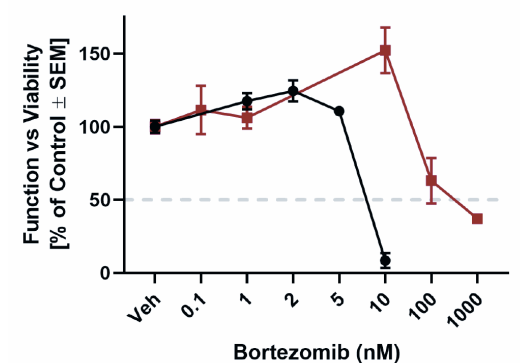

B

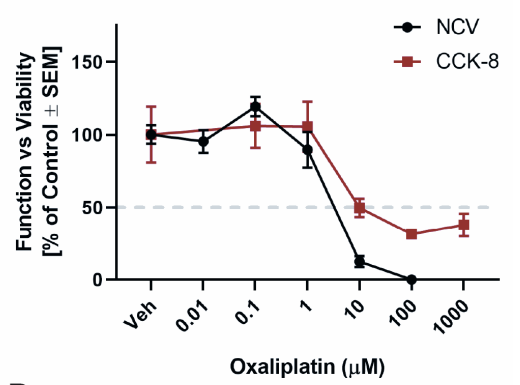

D

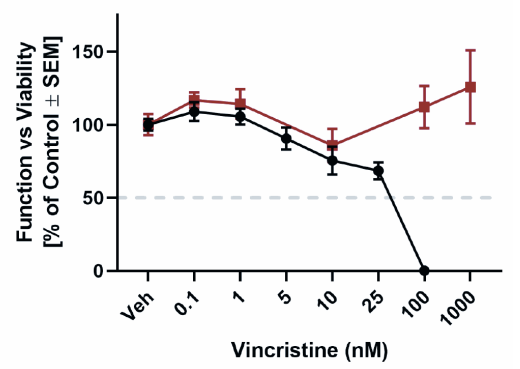

Fig. 4: Cell viability concentration response curves of nerve constructs exposed to chemotherapeutics with known neurotoxic effects (A-D) or compounds not known to induce neuropathy (E-F)

Cell viability measured using the CCK-8 cell viability assay (red circles) and the LDH cytotoxicity assay (blue squares). LDH data expressed as the cumulative absorbance of readings taken on day 2,4 , and 7 of drug exposure. $\mathrm{N}=6$; error bars represent SEM. *, $p$-value $\leq 0.05$ by one-way ANOVA followed by Dunnett's post-hoc test. Statistical comparisons made to vehicle control.
Fig. 5: Decreases in electrophysiological metrics occur before decreases in cell viability Concentration response curves of NCV (black circles) and CCK-8 cell viability (red squares) are replotted together from Figures 3 and 4 to demonstrate the difference in $\mathrm{IC}_{50}$ values. Dotted line is drawn at $50 \%$. 
(Fig. 4B,C). $10 \mathrm{nM} \mathrm{Bz}$ caused an initial increase in metabolic activity before it decreased at higher concentrations. This pattern was similar to the increase in NCV at a low Bz concentration. Ptx caused a decrease in metabolic activity to $78 \%$ of the control at the highest concentration $(10,000 \mathrm{nM})$, while Vn did not cause a statistically significant decrease in metabolic activity at any concentration employed (Fig. 4A,D).

The LDH assay revealed a concentration-dependent increase in plasma membrane damage and cytotoxicity across all drugs. Large increases in LDH release tended to occur at the same concentrations that caused decreases in metabolic activity determined with the CCK-8 assay. Drug concentrations resulting in dramatic increases in LDH were $200 \mathrm{nM}(\mathrm{Ptx}), 10 \mu \mathrm{M}(\mathrm{Ox})$, $100 \mathrm{nM}(\mathrm{Bz})$, and $1000 \mathrm{nM}(\mathrm{Vn})$. Minimal changes in cell viability metrics were found for constructs exposed to non-neuropathic acetaminophen and amoxicillin (Fig. 4E,F).
Tab. 1: Decreases in electrophysiological metrics occur before decreases in cell viability

\begin{tabular}{|l|l|l|l|}
\hline Drug & NCV IC $_{50}$ & AMP IC $_{50}$ & CCK-8 IC 50 \\
\hline Ptx & $245 \mathrm{nM}$ & $210 \mathrm{nM}$ & $>10,000 \mathrm{nM}$ \\
\hline Ox & $2.9 \mu \mathrm{M}$ & $8.4 \mu \mathrm{M}$ & $8.4 \mu \mathrm{M}$ \\
\hline $\mathrm{Bz}$ & $9.0 \mathrm{nM}$ & $9.1 \mathrm{nM}$ & $89 \mathrm{nM}$ \\
\hline $\mathrm{Vn}$ & $32 \mathrm{nM}$ & $20 \mathrm{nM}$ & $>1,000 \mathrm{nM}$ \\
\hline
\end{tabular}

$\mathrm{IC}_{50}$ values from nerve conduction velocity (NCV), amplitude (AMP) and CCK-8 cell viability dose response curves calculated using Graphpad Prism's four-parameter logistic curve model.
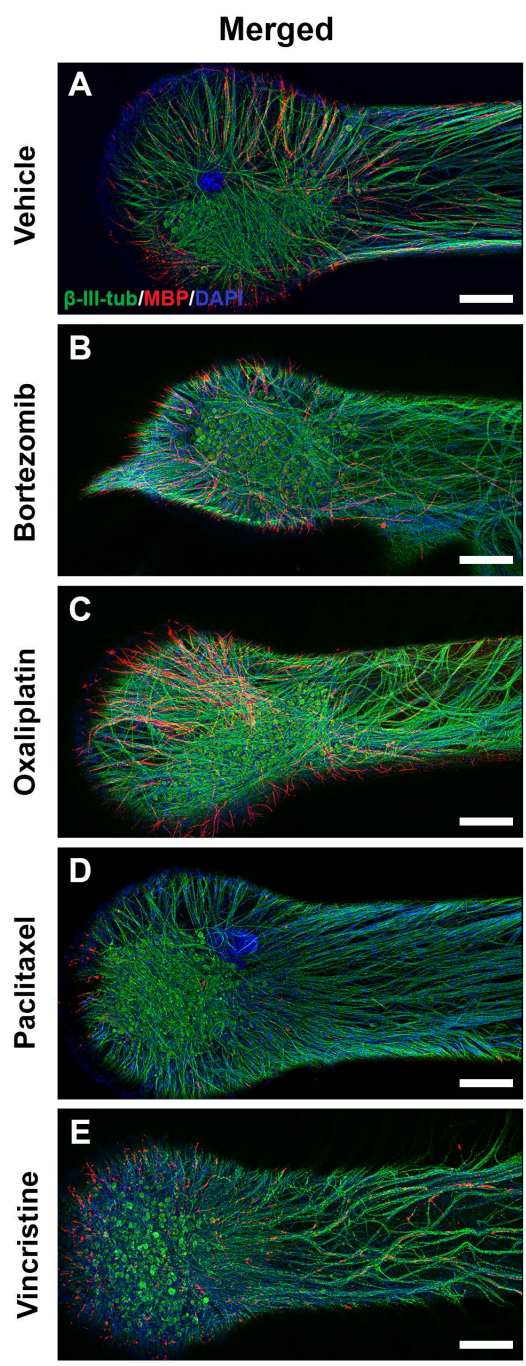

MBP
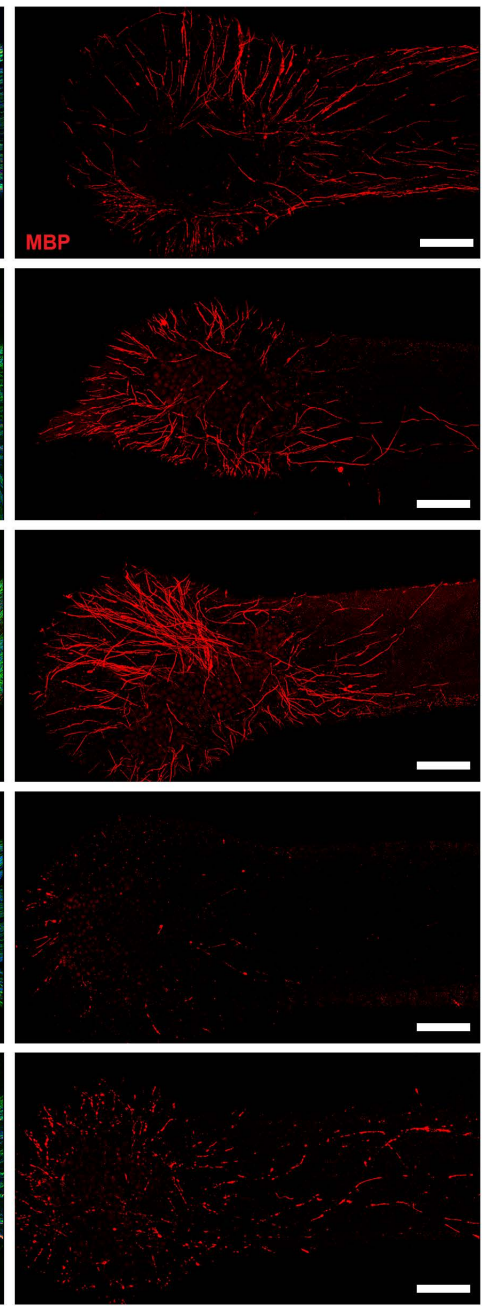

Fig. 6: Confocal micrographs of constructs reveal loss of myelin basic protein (MBP) only in response to $\mathrm{Vn}$ and Ptx treatment Confocal micrographs of constructs treated with $A$ ) vehicle control and NCV IC 50 concentrations of B) $\mathrm{Bz}(9 \mathrm{nM}), \mathrm{C})$ Ox $(2.9 \mu \mathrm{M}), \mathrm{D})$ Ptx (245 nM), and E) Vn (32 nM). Samples are labeled for MBP (red), $\beta$-III-tubulin (green), and DAPI (blue). Scale bar: $250 \mu \mathrm{m}$. Representative micrographs of $\mathrm{N}=2$ replicates. 


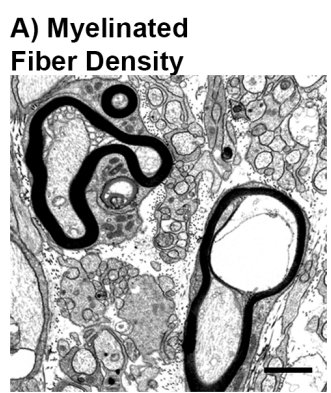

E) Eccentric Nuclei

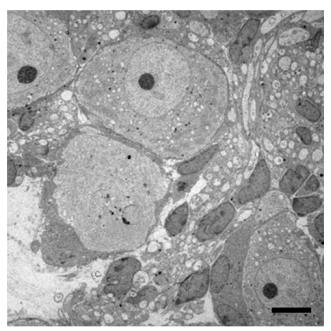

B) Nonmyelinated Fiber Density

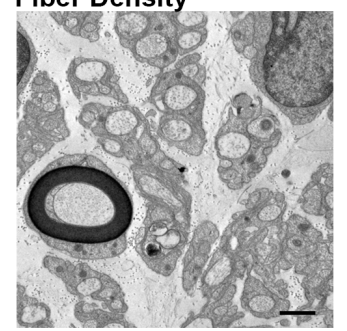

F) Degenerated Fibers

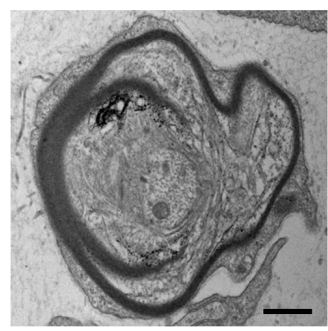

C) G-Ratio

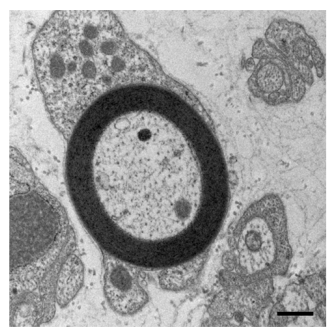

G) Nonmyelinated Fiber Diameter

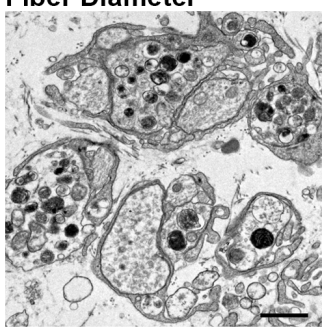

D) Irregular Myelin

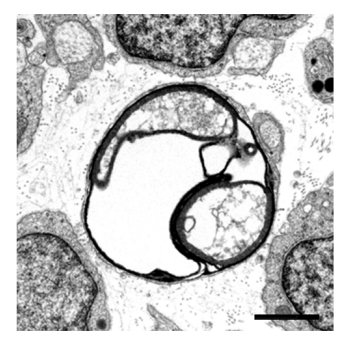

H) Myelinated

Fiber Diameter

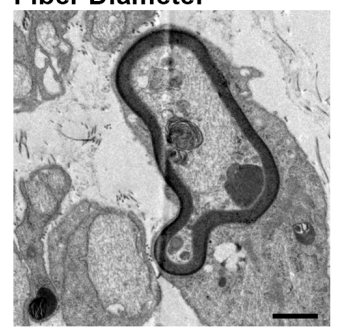

Fig. 7: Example micrographs of histomorphology metrics

A) A high density of myelinated axons used for measurement of density (Ox; scale bar: $1 \mu \mathrm{m})$. B) A moderately dense field of axons ensheathed by Remak Schwann cells. Larger caliber myelinated fiber also seen (Bz; scale bar: $1 \mu \mathrm{m}$ ). C) An optimum G-ratio and Schwann cell cytoplasm (vehicle; scale bar: $0.5 \mu \mathrm{m}$ ). D) Re-compacted myelin containing cytoplasm, a common type of irregular myelin present ( $\mathrm{Vn}$; scale bar: $1 \mu \mathrm{m})$. E) The fine structure of multiple neuronal cell bodies with eccentric nuclei (Bz; scale bar: $5 \mu \mathrm{m})$. F) A degenerated axon with disintegrating sheath (Vn; scale bar: $0.5 \mu \mathrm{m})$. G) Vincristine-treated axons are large and show frequent organelles and dystrophy (Vn; scale bar: $1 \mu \mathrm{m})$. H) Ptx-treated tissue shows dark, large-caliber myelinated axon-containing organelles and an oddly shaped sheath (Ptx; scale bar: $1 \mu \mathrm{m})$.

$\mathrm{IC}_{50}$ values for $\mathrm{NCV}$ and $\mathrm{CCK}-8$ cell viability were calculated using GraphPad Prism. IC 50 values for NCV were $245 \mathrm{nM}$ Ptx, $2.9 \mu \mathrm{M}$ Ox, $9 \mathrm{nM} \mathrm{Bz}$, and $32 \mathrm{nM} \mathrm{Vn}$. $\mathrm{IC}_{50}$ values for CCK-8 were > 10,000 nM Ptx, $8.4 \mu \mathrm{M}$ Ox, $89 \mathrm{nM} \mathrm{Bz}$, and > 1,000 nM $\mathrm{Vn}$ (Tab. 1). Comparison of the $\mathrm{IC}_{50}$ values demonstrates that decreases in NCV occurred at lower concentrations than decreases in cell viability. This suggests that decreases in NCV and AMP were not simply due to cell death, as the cells were still alive when the electrophysiological metrics began to decrease. This is visualized in Figure 5. For the following experiments (immunocytochemistry, mitochondrial membrane potential assay, and histology), chemotherapeutics at concentrations equal to the $\mathrm{NCV}$ $\mathrm{IC}_{50}$ values were administered to investigate the change in function, rather than viability, more closely.

\subsection{Chemotherapeutics induce damage to myelinated fibers}

In order to assess changes in myelin structure, immunocytochemistry and electron microscopy techniques were employed. A qualitative inspection revealed a decrease in myelin basic protein (MBP) expression in constructs exposed to chemotherapeutics at their respective $\mathrm{NCV} \mathrm{IC}_{50}$ values (Fig. 6). This effect was particularly prominent with $\mathrm{Vn}$ and Ptx. MBP is localized in the myelin sheath surrounding myelinated axons, and its importance to normal myelination suggests that extensive demyelination occurs in Vn and Ptx-induced peripheral neuropathy (Achiron and Miron, 2007; Deber and Reynolds, 1991). Analysis of TEM micrographs largely mirrored immunocytochemical findings. A decrease in myelinated fiber density was seen in all experimental groups. This was particularly appreciable in Vn and Px-treated samples, which additionally had a high frequency of myelin sheaths containing degenerated axons and a decreased G-ratio. Most sheaths in Ox- and Bz-treated samples were normally shaped and well-compacted (Fig. 7).

\subsection{RNoaC provides histopathological outcomes similar to in vivo results}

Transmission electron and light microscopy were employed to semi-quantitatively evaluate the fine structure of RNoaC constructs and to determine whether the $\mathrm{RNoaC}$ constructs recapitulate the histopathological hallmarks of in vivo CIPN models. The diameter of myelinated axons was slightly increased in all drug-treated $\mathrm{RNoaC}$ constructs, and this was particularly noticeable in Ptx-treated samples (Fig. 7H). Many of the myelinated axons appeared to be dystrophic and contained an accumulation of organelles. 
Tab. 2: Qualitative observations of increases and decreases in histometric measurements relative to vehicle controls

\begin{tabular}{|l|l|l|l|l|}
\cline { 2 - 5 } \multicolumn{1}{c|}{} & Ptx & Ox & Bz & Vn \\
\hline MBP staining & $\downarrow \downarrow \downarrow$ & - & - & $\downarrow \downarrow$ \\
\hline Myelinated fiber density & $\downarrow$ & $\downarrow$ & $\downarrow$ & $\downarrow \downarrow \downarrow$ \\
\hline Nonmyelinated fiber density & $\downarrow \downarrow$ & $\downarrow$ & $\downarrow$ & $\downarrow \downarrow \downarrow$ \\
\hline G-ratio & - & - & - & $\downarrow \downarrow \downarrow$ \\
\hline \% Irregular myelin & $\uparrow$ & $\uparrow$ & - & $\uparrow$ \\
\hline \% Eccentric nuclei & $\uparrow \uparrow \uparrow$ & $\uparrow \uparrow \uparrow$ & $\uparrow \uparrow$ & $\uparrow$ \\
\hline$\%$ Degenerated fibers & $\uparrow$ & - & - & $\uparrow \uparrow \uparrow$ \\
\hline Nonmyelinated fiber diameter & $\uparrow \uparrow \uparrow$ & $\uparrow$ & $\uparrow \uparrow \uparrow$ & $\uparrow$ \\
\hline Myelinated fiber diameter & $\uparrow \uparrow$ & - & $\uparrow$ & - \\
\hline
\end{tabular}

"-" indicates similar values. Three arrows indicate dramatic, readily apparent deviations from vehicle controls. Two arrows point to a moderately appreciable deviation, while one arrow shows a trend away from control values.

Diameters of nonmyelinated fibers were similar to the vehicle control for Ox-treated $\mathrm{RNoaC}$, whereas larger diameters were consistently observed in Ptx, Bz, and Vn conditions (Fig. 7H). Nonmyelinated fiber density largely followed the same trend as myelinated fiber diameter, with Vn and Ptx exposure causing large decreases in density compared to vehicle control, while $\mathrm{Ox}$ and Bz caused lesser decreases.

Nuclear eccentricity was evaluated by light microscopy analysis of RNoaC cross-sections stained with toluidine blue. Nuclear eccentricity is known to occur as a result of axotomy and has been investigated in numerous in vivo models of CIPN (Lieberman, 1971; Holmes et al., 1998; Jamieson et al., 2007). All conditions treated with $\mathrm{Ptx}, \mathrm{Ox}$ and $\mathrm{Bz}$ displayed an elevated rate of eccentric nuclei compared to vehicle control (Fig. 7E). Vn-treated constructs lacked such changes. However, numerous nuclei exhibited condensation and margination of chromatin and were likely engaged in apoptotic cell death. A summary of the observations is tabulated in Table 2 .

\subsection{Mitochondrial health is compromised with increasing drug concentration}

The RNoaC not only provides clinically relevant metrics but can also be used for common 2D assays such as examining mitochondrial membrane potential. The TMRE Mitochondrial Membrane Potential Assay was used to evaluate mitochondrial health of the RNoaC constructs. After 7 days of drug administration, the live cells were stained with TMRE dye, a positively charged fluorescent dye that detects the negative charge across healthy mitochondrial membranes. The axonal region in the $\mathrm{RNoaC}$ channel of the vehicle control displayed bright fluorescent staining compared to FCCP, the positive control, when imaged with the same exposure. The fluorescent signal was noticeably diminished in the channel region of constructs exposed to $100 \mathrm{nM} \mathrm{Bz}$ or $100 \mu \mathrm{M} \mathrm{Ox}$, indicating that mitochondrial heath was compromised (Fig. 8A). This was confirmed using a custom Image $J$ macro to calculate the mean pixel intensity of thresholded images to represent the level of fluorescence in the images (Fig. 8B). There was also a significant decrease in fluorescent intensity between $2.9 \mu \mathrm{M}$ and $100 \mu \mathrm{M}$ Ox. The mean fluorescent/pixel intensity of the remaining constructs was not significantly different from the vehicle control.

Images taken of the DRG somal region in the RNoaC constructs revealed a similar pattern of mitochondrial membrane potential. There was little difference in staining after Ptx and Vn treatment compared to vehicle control, however Bz and Ox, especially at high concentrations, dampened the fluorescent signal (Fig. S1 $\mathrm{A}^{1}$ ). While the calculated mean pixel intensities confirmed the trend towards decreasing mean fluorescent intensity in $\mathrm{Ox}$ and $\mathrm{Bz}$ treated constructions at higher concentrations, the difference was not statistically significant (Fig. S1B ${ }^{1}$ ).

\section{Discussion}

There is a need to develop predictive in vitro platforms that recapitulate key features of neuropathy and can reliably predict clinically relevant metrics of toxicity for preclinical screens (Pollard et al., 2019). Organ-on-a-chip systems satisfy this need by presenting a more physiologically accurate and dynamic 3D system compared to traditional in vitro models, while providing more control of parameters than in vivo animal models. In this study, we used our previously developed rat nerve-on-a-chip (Huval et al., 2015; Khoshakhlagh et al., 2018) with its unique ability to capture clinically relevant electrophysiological metrics in a model of CIPN.

In vitro models of neurotoxicity use primary DRGs, cell lines or differentiated stem cells to study the mechanisms underlying drug-induced neurotoxicity (Jones et al., 2018; Fukuda and Segal, 2017; Rana et al., 2017). Common assays for in vitro mechanistic studies include cellular viability, calcium imaging, and neurite outgrowth measurements (Fukuda and Segal, 2017; 
A
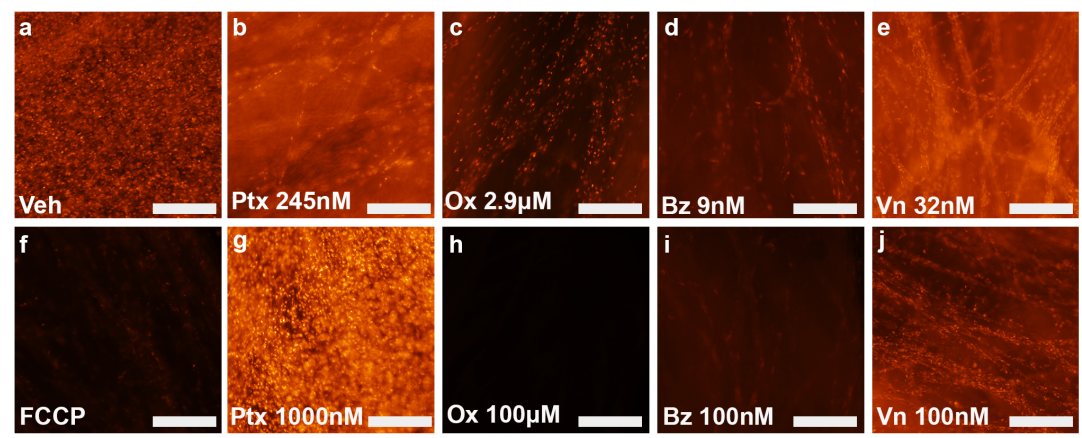

Fiber tract

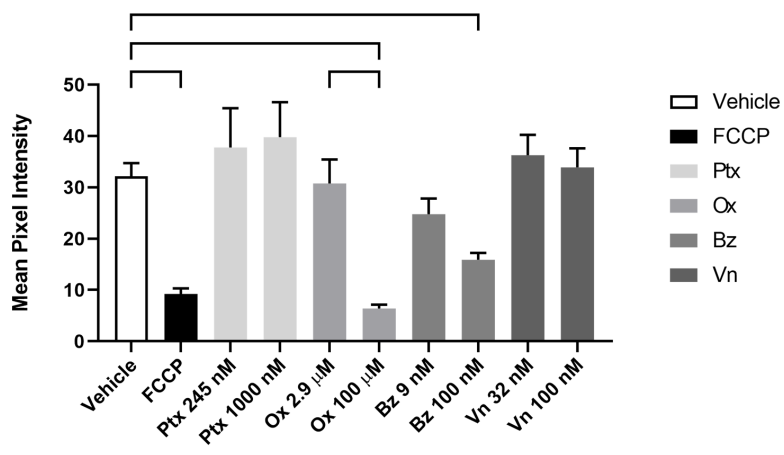

Fig. 8: Changes in mitochondrial membrane polarization in axon extensions after exposure to drugs A) Live cells stained with TMRE MMP dye demonstrate changes in mitochondrial membrane polarization after exposure to vehicle control (a), Ptx (b, g), Ox (c, h), Bz (d, i), $\mathrm{Vn}(\mathrm{e}, \mathrm{j})$ or positive control FCCP (f). Representative images of axon extensions. Scale bar: $100 \mu \mathrm{m}$. B) Mean pixel intensity calculated from $\mathrm{N}=4$ samples with images taken from 3 random fields of view. Error bars represent SEM. Brackets indicate $p$-value $\leq 0.05$ by one-way ANOVA followed by Dunnett's post-hoc test.
Brewer et al., 2016; Golden and Johnson, 2004). In addition, multielectrode arrays are used to measure the generation and propagation of spontaneous or evoked electrical activity (Gribi et al., 2018). 2D in vitro systems are limited in that they do not replicate peripheral nervous system architecture and thus may not be representative of the in vivo condition.

The standard endpoint in animal studies is histopathological assessment of nervous tissue, which focuses on degeneration of myelin, neurons and axons, but has limited translatability to humans (Li et al., 2013; Höke and Ray, 2014). Better translatable studies are behavioral studies and nerve conduction studies, in which animals demonstrate neurophysiological changes similar to human disease. Nerve conduction studies, which measure the amplitude and conduction of CAPs, are particularly translatable, as these are the gold standard in the clinical setting for evaluating CIPN (Brewer et al., 2016).

Our RNoaC constructs are unique in that they provide characteristic output metrics of both in vitro and in vivo studies. The constructs exhibit electrophysiological abnormalities similar to those seen in both human and rat CIPN. We found a decrease in measured CAP NCV and AMP with drug exposure, a phenomenon observed in other preclinical models and in the clinical setting (Leandri et al., 2012; Gilardini et al., 2012; Wozniak et al., 2018; Authier et al., 2003). In addition to recapitulating in vivo neuropathy, our model provides a clinically relevant screen for neurotoxicity, as CIPN is assessed in the clinical oncology setting using nerve conduction studies (Brewer et al., 2016).
The NCV measured in our RNoaC typically ranges between $0.2-0.5 \mathrm{~m} / \mathrm{s}$ when electrically stimulating the axons and recording action potentials at the soma at a distance of 1-4 $\mathrm{mm}$. These velocities are lower than the average NCV of $30-60 \mathrm{~m} / \mathrm{s}$ found in motor and sensory nerves in rats (Thomas et al., 1981; Zotova and Arezzo, 2013). However, such values were collected from recording large myelinated fibers of adult animals. When measuring unmyelinated C-fibers in rats, velocities were found to be $1-2 \mathrm{~m} / \mathrm{s}$ (Zotova and Arezzo, 2013). Furthermore, younger animals are known to exhibit slower conduction velocities, presumably due to incomplete development of the nerves (Hort-Legrand et al., 2006; Stanley, 1981). An additional property besides myelination that affects the biophysics of nerve conduction is fiber diameter. Our model contains both myelinated and unmyelinated fibers with a typical diameter of 1-1.5 $\mu \mathrm{m}$ from dorsal root neurons collected from embryonic rats and recorded at room temperature, so it is not surprising that the NCV are slower in our model.

Concentration-response curves of chemotherapeutics in electrically stimulated neurons showed significant decreases in $\mathrm{NCV}$ and AMP occurring before significant decreases in cell viability as measured with CCK-8 and LDH colorimetric assays. This demonstrates that functional measurements represent a more sensitive metric, and that the model allows distinctions to be made between electrical functional deficits and general cytotoxicity.

The most common assay for assessing electrical function in vitro is patch-clamp electrophysiology, which measures the electri- 
cal properties of ion channels (Gauchan et al., 2009) and neuron membranes (Liu et al., 2002). The patch-clamp technique is limited in that it can only assess individual neurons rather than the dynamics of neuronal populations (Accardi et al., 2016). The microelectrode array (MEA) is a method of extracellular recording that addresses this issue by sensing extracellular field potentials in population-based analysis (Yang et al., 2016). MEAs are used in neuropathic pain studies to measure the spontaneous firing rate of dissociated DRGs (Newberry et al., 2016; Yang et al., 2016) but typically do not provide clinically analogous information when recording dissociated cells cultured on planar substrates. The advantage of our electrophysiology method is that we can measure NCV and AMP from evoked CAPs in a manner similar to clinical methods in humans and animals. Our technique is similar, yet not identical, as we record from the DRG body to facilitate recording and prevent overly damaging axonal structures, whereas the accepted clinical technique stimulates axons and records at two locations both along the axons (Cummins et al., 1979). We are currently working on the incorporation of integrated microelectrodes, which will greatly facilitate more accurate measurements of NCV and will more easily allow recording at multiple locations including along the axons. Although the neuron culture within our RNoaC constructs is dense enough to measure CAP, the axons are not fully fasciculated at the microscopic level, and we are working to reduce the channel width in order to reduce the distance between the fibers. Alternative approaches to study the fasciculated networks within our RNoaC constructs include voltage sensitive dyes.

An interesting phenomenon we observed is an initial increase in NCV at low concentrations of Ptx and Bz. Other studies have attributed this phenomenon to increased poration of membranes, leading to hyperexcitability that has been observed at low, pre-toxic concentrations of anti-cancer drugs (Zhang and Dougherty, 2014; Wing et al., 2017). There was a similar increase in metabolic activity at low concentrations of $\mathrm{Bz}$, once again corresponding to in vivo experiments in which low doses of anti-cancer drugs induced increases in metabolic activity before activity reduced at higher concentrations (Li et al., 2016).

A reduction in NCV is typically associated with a reduction of myelin. However, it is unclear to what extent NCV slowing is due to demyelination or degeneration of peripheral nerve axons, as many studies have reported decreased NCV and onset of CIPN without or with only mild changes to the myelin structure (Starobova and Vetter, 2017; Gilardini et al., 2012; Meregalli et al., 2018; Duggett and Flatters, 2017). It is unknown what mechanism produces a slowed NCV in the absence of demyelination. In our RNoaC platform, all chemotherapeutics induced a slowed $\mathrm{NCV}$, but the presence of myelin-related pathologies appeared to be drug-dependent. We saw the greatest changes to myelin structure in Ptx and Vn-treated constructs as visualized with immunostaining and TEM. This finding corresponded well with in vivo rat studies in which "degeneration of distal sensory axons, secondary demyelination, and nerve fiber loss" was reported in Ptx and Vn-induced neuropathies (Starobova and Vetter, 2017). Our finding of minimal myelin damage upon $\mathrm{Bz}$ and $\mathrm{Ox}$ treatment also corresponds with in vivo results. Gilardini et al. (2012) did not see changes in myelin sheath structure of Bz-treated rats as analyzed with electron microscopy and X-ray diffraction. Cavaletti et al. (2001) observed mild changes in rat sciatic nerve and DRG after Ox treatment with the presence of normally-myelinated Schwann cells, and rarely axons undergoing degeneration. Amplitude, the other metric used to assess neuronal functionality in the RNoaC in vitro model, is often shown to decrease when various processes of axonal degeneration are underway (Chung et al., 2014; Boehmerle et al., 2015; Leandri et al., 2012). Axonal degeneration as the cause for slowed AMP in our RNoaC is supported by the elevated number of myelin sheaths containing degenerated axons. Nearly every sheath seen in Vn-treated constructs contained a degenerated axon while this metric approached $25 \%$ for Ptx.

Taken together, the histological data collected from our RNoaCs correlates with past in vivo histomorphometry and the results of our other assays, suggesting its application as a relevant model of CIPN. However, it is important to note that histomorphic analysis was performed on only a few samples and was therefore not statistically powerful. Future work will include a more rigorous analysis of $\mathrm{RNoaC}$ histological samples.

Anti-cancer drugs are known to induce mitochondrial damage through varying mechanisms, leading to impairment of ATPase-dependent $\mathrm{Na} / \mathrm{K}$ pumps and calcium homeostasis alterations (Canta et al., 2015; Cheng et al., 2015). Our nerve constructs exhibited changes in mitochondrial membrane polarization, suggesting that mitochondrial dysfunction is involved in the neuropathy of the constructs. As expected, the mitochondrial results corroborated the findings of the CCK-8 cell metabolic assay: At high concentrations of $\mathrm{Bz}$ and $\mathrm{Ox}$ there was a decrease in both the level of TMRE fluorescent intensity and CCK-8 values, while Vn and Ptx did not cause significant decreases. Therefore, the nerve constructs are compatible with kit-based colorimetric and fluorescent imaging methods to analyze cell viability. Additionally, the TMRE mitochondrial assay allowed us to look at toxicity in specific regions of the $\mathrm{RNoaC}$ construct, namely in the channel or bulb regions. In this study, we used the CCK8 assay to focus on the neuronal cell bodies in the bulb region. Performing both assays allowed us to both confirm the results against each other and to focus on cell viability within certain tissue regions. Interestingly, the TMRE mitochondrial assay did not reveal significant mitochondrial dysfunction upon Ptx treatment although other studies have suggested mitotoxicity as underlying Ptx CIPN (Flatters and Bennett, 2006). It is possible that the presence of other cell types in our culture that do not lose mitochondrial function when exposed to Ptx, such as Schwann cells (Imai et al., 2017), mitigates the changes seen in the TMRE assay. Cell-type specific mitotoxicity could be investigated at higher magnification as colocalization with antibody staining in future studies.

As our model represents the structure and function of peripheral nerves, there are similarities in anatomy and biophysics. However, our model is not equivalent in every respect, as it lacks distinct fascicles, axons up to $1 \mathrm{~m}$ long, multiple different fiber types and diameters, etc. Consequently, our model does not recapitulate every aspect of peripheral nerve anatomy and biophys- 
ics, but it does capture some key features that make it useful as a functional model able to distinguish different forms of toxicity.

An interesting observation is that the four tested chemotherapeutics had differing effects on the analyzed metrics. At the NCV $\mathrm{IC}_{50}$ concentration, samples treated with $\mathrm{Ptx}$ and $\mathrm{Vn}$ induced a more dramatic change in myelin sheaths as seen by decreased MBP staining and histological metrics including myelinated fiber density and G-ratio. While there were no observable changes in mitochondrial health at the $\mathrm{NCV} \mathrm{IC}_{50}$ concentrations, higher concentrations of $\mathrm{Bz}$ and $\mathrm{Ox}$ compromised mitochondrial health. A summary of all metrics is tabulated in Table $\mathrm{S} 2{ }^{1}$.

It is possible that the differing responses to the chemotherapeutics are due to their varying mechanisms of action. It is known that Ptx disrupts the assembly of microtubule subunits during cell division. While it is unknown how exactly Ptx causes neuropathy, it is thought that this is due to dying-back axonopathy. Vn, which destabilizes microtubule formation, causes a similar pathophysiology (Staff et al., 2017). In our nerve constructs, Ptx and Vn treated constructs had the highest relative increase of degenerated fibers and the greatest decrease in fiber density, suggesting that our platform reflects the specific pathophysiology of these compounds. $\mathrm{Bz}$ is a proteasome inhibitor and appears to be neurotoxic due to interference with mitochondrial function. Ox damages DRG neurons by forming adducts with nuclear and mitochondrial DNA (Staff et al., 2017). Unlike in the nucleus, the mitochondria do not have a DNA repair process and damaged protein accumulates. In line, only Bz- and Ox-exposed constructs showed compromised mitochondrial health in the TMRE assay.

DRGs from Long-Evans rats were used to maintain continuity with our previous work (Huval et al., 2015; Khoshakhlagh et al., 2018), though in order to facilitate direct comparisons with historical toxicology studies, it may be helpful to perform future studies with cells from strains more commonly used in toxicology research. We have recently created a similar platform comprised of human induced pluripotent stem cell (iPSC)-derived neurons and primary human Schwann cells (Sharma et al., 2019) that we will continue to develop in order to further reduce the use of animals and improve clinical relevance. iPSC-derived neurons are generally considered phenotypically immature and have distinct genomic profiles that will need to be accounted for in the transition to human cell-based systems. Furthermore, serum-free culture of rat DRGs is feasible (Anderson et al., 2018; George et al., 2019), and we are currently making the transition to serum-free culture.

In this study, we present a model of CIPN using our rat nerveon-a-chip construct. The dual hydrogel construct directs cell growth into nerve-like bundles and is unique in that it provides conventional electrophysiological and histological data. These metrics are the current gold standards for functional and structural endpoints and are the preferred metrics for assessing neuropathological conditions. Additionally, our RNoaC is compatible with standard kit assays for cell viability and mitochondrial health. RNoaC constructs are sensitive to CIPN, as analyzed by electrophysiology and histology, and show the hallmarks of in vivo CIPN. The ability to collect clinically relevant data from this $\mathrm{RNoaC}$ platform suggests its use as an effective tool for screen- ing potentially harmful drugs and other neuropathy-inducing agents. The use of in vitro platforms that can provide in vivo- and clinical-like data, such as the $\mathrm{RNoaC}$, has a strong potential to reduce the use of animals in pre-clinical studies. We plan to build on these rat and human cell-based systems by studying further types of neuropathology and screening therapeutic molecules to further demonstrate the abilities of our RNoaC platform.

\section{References}

Accardi, M. V., Pugsley, M. K., Forster, R. et al. (2016). The emerging role of in vitro electrophysiological methods in CNS safety pharmacology. J Pharmacol Toxicol Methods 81, 47-59. doi:10.1016/j.vascn.2016.03.008

Achiron, A. and Miron, S. (2007). Myelin associated antibodies: Myelin-associated glycoprotein autoantibodies and myelin proteolipid autoantibodies in neurologic diseases. In Y. Shoenfeld, M. E. Gershwin and P. L. Meroni (eds.), Autoantibodies (619-626). $2^{\text {nd }}$ edition. Elsevier Science. doi:10.1016/B978044452763-9/50080-9

Anderson, W. A., Willenberg, A. R., Bosak, A. J. et al. (2018). Use of a capillary alginate gel (Capgel $\left.{ }^{\mathrm{TM}}\right)$ to study the three-dimensional development of sensory nerves reveals the formation of a rudimentary perineurium. J Neurosci Methods 305, 46-53. doi:10.1016/J.JNEUMETH.2018.05.003

Argyriou, A. A., Bruna, J., Marmiroli, P. et al. (2012). Chemotherapy-induced peripheral neurotoxicity (CIPN): An update. Crit Rev Oncol Hematol 82, 51-77. doi:10.1016/J.Critrevonc. 2011.04.012

Authier, N., Gillet, J.-P., Fialip, J. et al. (2003). A new animal model of vincristine-induced nociceptive peripheral neuropathy. Neurotoxicology 24, 797-805. doi:10.1016/S0161-813X (03)00043-3

Boehmerle, W., Huehnchen, P., Peruzzaro, S. et al. (2015). Electrophysiological, behavioral and histological characterization of paclitaxel, cisplatin, vincristine and bortezomib-induced neuropathy in C57Bl/6 mice. Sci Rep 4, 6370. doi:10.1038/ srep06370

Bowser, D. A. and Moore, M. J. (2019). Biofabrication of neural microphysiological systems using magnetic spheroid bioprinting. Biofabrication 12, 015002. doi:10.1088/1758-5090/ ab41b4

Brewer, J. R., Morrison, G., Dolan, M. E. et al. (2016). Chemotherapy-induced peripheral neuropathy: Current status and progress. Gynecol Oncol 140, 176-183. doi:10.1016/j.ygyno. 2015.11.011

Canta, A., Pozzi, E. and Carozzi, V. A. (2015). Mitochondrial dysfunction in chemotherapy-induced peripheral neuropathy (CIPN). Toxics 3, 198-223. doi:10.3390/toxics3020198

Cavaletti, G., Tredici, G., Petruccioli, M. et al. (2001). Effects of different schedules of oxaliplatin treatment on the peripheral nervous system of the rat. Eur J Cancer 37, 2457-2463. doi:10.1016/S0959-8049(01)00300-8

Cavaletti, G. and Marmiroli, P. (2010). Chemotherapy-induced peripheral neurotoxicity. Nat Rev Neurol 6, 657-666. doi:10.1038/nrneurol.2010.160 
Cheng, X. L., Liu, H. Q., Wang, Q. et al. (2015). Chemotherapy-induced peripheral neurotoxicity and complementary and alternative medicines: Progress and perspective. Front Pharmacol 6, 234. doi:10.3389/fphar.2015.00234

Chung, T., Prasad, K. and Lloyd, T. E. (2014). Peripheral neuropathy. Neuroimaging Clin N Am 24, 49-65. doi:10.1016/j. nic.2013.03.023

Cummins, K. L., Perkel, D. H. and Dorfman, L. J. (1979). Nerve fiber conduction-velocity distributions. I. Estimation based on the single-fiber and compound action potentials. Electroencephalogr Clin Neurophysiol 46, 634-646. doi:10.1016/00134694(79)90101-9

Curley, J. L. and Moore, M. J. (2011). Facile micropatterning of dual hydrogel systems for 3D models of neurite outgrowth. $J$ Biomed Mater Res A 99, 532-543. doi:10.1002/jbm.a.33195

Deber, C. M. and Reynolds, S. J. (1991). Central nervous system myelin: Structure, function, and pathology. Clin Biochem 24, 113-134. doi:10.1016/0009-9120(91)90421-A

Duggett, N. A. and Flatters, S. J. L. (2017). Characterization of a rat model of bortezomib-induced painful neuropathy. $\mathrm{Br} J$ Pharmacol 174, 4812-4825. doi:10.1111/bph.14063

Flatters, S. J. and Bennett, G. J. (2006). Studies of peripheral sensory nerves in paclitaxel-induced painful peripheral neuropathy: Evidence for mitochondrial dysfunction. Pain 122, 245257. doi:10.1016/j.pain.2006.01.037

Frank, C., Himmelstein, D. U., Woolhandler, S. et al. (2014). Era of faster FDA drug approval has also seen increased black-box warnings and market withdrawals. Health Aff 33, 1453-1459. doi:10.1377/hlthaff.2014.0122

Fukuda, Y., Li, Y. and Segal, R. A. (2017). A mechanistic understanding of axon degeneration in chemotherapy-induced peripheral neuropathy. Front Neurosci 11, 481. doi:10.3389/ fnins.2017.00481

Gauchan, P., Andoh, T., Ikeda, K. et al. (2009). Mechanical allodynia induced by paclitaxel, oxaliplatin and vincristine: Different effectiveness of gabapentin and different expression of voltage-dependent calcium channel $\alpha 2 \delta$-1 subunit. Biol Pharm Bull 32, 732-734. doi:10.1248/bpb.32.732

George, D. S., Anderson, W. A., Sommerhage, F. et al. (2019). Bundling of axons through a capillary alginate gel enhances the detection of axonal action potentials using microelectrode arrays. J Tissue Eng Regen Med 13, 385-395. doi:10.1002/ term. 2793

Ghaemmaghami, A. M., Hancock, M. J., Harrington, H. et al. (2012). Biomimetic tissues on a chip for drug discovery. Drug Discov Today 17, 173-181. doi:10.1016/j.drudis.2011.10.029

Gilardini, A., Avila, R. L., Oggioni, N. et al. (2012). Myelin structure is unaltered in chemotherapy-induced peripheral neuropathy. Neurotoxicology 33, 1-7. doi:10.1016/J.Neuro. 2011.10.010

Golden, J. P. and Johnson, E. M. (2004). Models of chemotherapy drug-induced peripheral neuropathy. Drug Discov Today Dis Models 1, 186-191. doi:10.1016/j.ddmod.2004.09.005

Gribi, S., du Bois de Dunilac, S., Ghezzi, D. et al. (2018). A microfabricated nerve-on-a-chip platform for rapid assessment of neural conduction in explanted peripheral nerve fibers. Nat
Commun 9, 4403. doi:10.1038/s41467-018-06895-7

Höke, A. (2012). Animal models of peripheral neuropathies. Neurotherapeutics 9, 262-269. doi:10.1007/s13311-012-0116-y

Höke, A. and Ray, M. (2014). Rodent models of chemotherapy-induced peripheral neuropathy. ILAR J 54, 273-281. doi:10. 1093/ilar/ilt053

Holmes, J., Stanko, J., Varchenko, M. et al. (1998). Comparative neurotoxicity of oxaliplatin, cisplatin, and ormaplatin in a Wistar rat model. Toxicol Sci 46, 342-351. doi:10.1006/ toxs. 1998.2558

Hort-Legrand, C., Noah, L., Mériguet, E. et al. (2006). Motor and sensory nerve conduction velocities in Yucatan minipigs. Lab Anim 40, 53-57. doi:10.1258/002367706775404345

Huval, R. M., Miller, O. H., Curley, J. L. et al. (2015). Microengineered peripheral nerve-on-a-chip for preclinical physiological testing. Lab Chip 15, 2221-2232. doi:10.1039/ C4LC01513D

Imai, S., Koyanagi, M., Azimi, Z. et al. (2017). Taxanes and platinum derivatives impair Schwann cells via distinct mechanisms. Sci Rep 7, 5947. doi:10.1038/s41598-017-05784-1

Jamieson, S., Liu, J., Connor, B. et al. (2007). Nucleolar enlargement, nuclear eccentricity and altered cell body immunostaining characteristics of large-sized sensory neurons following treatment of rats with paclitaxel. Neurotoxicology 28, 10921098. doi:10.1016/j.neuro.2007.04.009

Jones, I., Yelhekar, T. D., Wiberg, R. et al. (2018). Development and validation of an in vitro model system to study peripheral sensory neuron development and injury. Sci Rep 8, 15961. doi:10.1038/s41598-018-34280-3

Kapałczyńska, M., Kolenda, T., Przybyła, W. et al. (2018). 2D and 3D cell cultures - A comparison of different types of cancer cell cultures. Arch Med Sci 14, 910-919. doi:10.5114/ AOMS.2016.63743

Khoshakhlagh, P., Sivakumar, A., Pace, L. A. et al. (2018). Methods for fabrication and evaluation of a 3D microengineered model of myelinated peripheral nerve. J Neural Eng 15, 064001. doi:10.1088/1741-2552/aae129

Kimlin, L., Kassis, J. and Virador, V. (2013). 3D in vitro tissue models and their potential for drug screening. Expert Opin Drug Discov 8, 1455-1466. doi:10.1517/17460441.2013.852 181

Leandri, M., Ghignotti, M., Emionite, L. et al. (2012). Electrophysiological features of the mouse tail nerves and their changes in chemotherapy induced peripheral neuropathy (CIPN). J Neurosci Methods 209, 403-409. doi:10.1016/J. JNEUMETH.2012.07.005

Li, C., Bunner, A. E. and Pippin, J. J. (2013). From animal models to clinical practicality: Lessons learned from current translational progress of diabetic peripheral neuropathy. In $\mathrm{N}$. Souayah (ed.), Peripheral Neuropathy - A New Insight into the Mechanism, Evaluation and Management of a Complex Disor$\operatorname{der}$ (Chapter 2). InTech. doi:10.5772/55364

Li, Q., Ma, Z., Liu, Y. et al. (2016). Low doses of paclitaxel enhance liver metastasis of breast cancer cells in the mouse model. FEBS J 283, 2836-2852. doi:10.1111/febs. 13767

Lieberman, A. R. (1971). The axon reaction: A review of the prin- 
cipal features of perikaryal responses to axon injury. Int Rev Neurobiol 14, 49-124. doi:10.1016/S0074-7742(08)60183-X

Liu, C. N., Devor, M., Waxman S. G. et al. (2002). Subthreshold oscillations induced by spinal nerve injury in dissociated muscle and cutaneous afferents of mouse DRG. J Neurophysiol 87, 2009-2017. doi:10.1152/jn.00705.2001

Manji, H. (2013). Drug-induced neuropathies. Handb Clin Neurol 115, 729-742. doi:10.1016/B978-0-444-52902-2.00042-4

Meregalli, C., Marjanovic, I., Scali, C. et al. (2018). High-dose intravenous immunoglobulins reduce nerve macrophage infiltration and the severity of bortezomib-induced peripheral neurotoxicity in rats. $J$ Neuroinflammation 15, 232. doi:10.1186/ s12974-018-1270-x

Newberry, K., Wang, S., Hoque, N. et al. (2016). Development of a spontaneously active dorsal root ganglia assay using multiwell multielectrode arrays. J Neurophysiol 115, 3217-3228. doi:10.1152/jn.01122.2015

Onakpoya, I. J., Heneghan, C. J. and Aronson, J. K. (2016). Post-marketing withdrawal of 462 medicinal products because of adverse drug reactions: A systematic review of the world literature. BMC Med 14, 10. doi:10.1186/s12916-016-0553-2

Peters, C. M., Jimenez-Andrade, J. M., Kuskowski, M. A. et al. (2007). An evolving cellular pathology occurs in dorsal root ganglia, peripheral nerve and spinal cord following intravenous administration of paclitaxel in the rat. Brain Res 1168, 46-59. doi:10.1016/j.brainres.2007.06.066

Pollard, K. J., Sharma, A. D. and Moore, M. J. (2019). Neural microphysiological systems for in vitro modeling of peripheral nervous system disorders. Bioelectron Med 2, 101-117. doi:10.2217/bem-2019-0018

Rana, P., Luerman, G., Hess, D. et al. (2017). Utilization of iP$\mathrm{SC}$-derived human neurons for high-throughput drug-induced peripheral neuropathy screening. Toxicol In Vitro 45, 111-118. doi:10.1016/J.TIV.2017.08.014

Rivera, E. and Cianfrocca, M. (2015). Overview of neuropathy associated with taxanes for the treatment of metastatic breast cancer. Cancer Chemother Pharmacol 75, 659-670. doi:10. 1007/s00280-014-2607-5

Sharma, A. D., McCoy, L., Jacobs, E. et al. (2019). Engineering a $3 \mathrm{D}$ functional human peripheral nerve in vitro using the nerveon-a-chip platform. Sci Rep 9, 8921. doi:10.1038/s41598-01945407-5

Staff, N. P., Grisold, A., Grisold, W. et al. (2017). Chemotherapy-induced peripheral neuropathy: A current review. Ann Neurol 81, 772-781. doi:10.1002/ANA.24951
Stanley, E. F. (1981). Sensory and motor nerve conduction velocities and the latency of the $\mathrm{H}$ reflex during growth of the rat. Exp Neurol 71, 497-506. doi:10.1016/0014-4886(81)90027-3

Starobova, H. and Vetter, I. (2017). Pathophysiology of chemotherapy-induced peripheral neuropathy. Front Mol Neurosci 10, 174. doi:10.3389/fnmol.2017.00174

Thomas, D. (2016). Clinical Development Success Rates 20062015. San Diego. https://bit.ly/36hfzv9 (accessed 18.05.2020).

Thomas, P. K., Jefferys, J. G., Sharma, A. K. et al. (1981). Nerve conduction velocity in experimental diabetes in the rat and rabbit. J Neurol Neurosurg Psychiatry 44, 233-238. doi:10.1136/ jnnp.44.3.233

Wing, C., Komatsu, M., Delaney, S. et al. (2017). Application of stem cell derived neuronal cells to evaluate neurotoxic chemotherapy. Stem Cell Res 22, 79-88. doi:10.1016/J. SCR.2017.06.006

Wozniak, K. M., Vornov, J. J., Wu, Y. et al. (2018). Peripheral neuropathy induced by microtubule-targeted chemotherapies: Insights into acute injury and long-term recovery. Cancer Res 78, 817-829. doi:10.1158/0008-5472.CAN-17-1467

Yang, Y., Huang, J., Mis, M. A. et al. (2016). Nav1.7-A1632G mutation from a family with inherited erythromelalgia: Enhanced firing of dorsal root ganglia neurons evoked by thermal stimuli. JNeurosci 36, 7511-7522. doi:/10.1523/JNEUROSCI. 0462-16.2016

Zhang, H. and Dougherty, P. M. (2014). Enhanced excitability of primary sensory neurons and altered gene expression of neuronal ion channels in dorsal root ganglion in paclitaxel-induced peripheral neuropathy. Anesthesiology 120, 1463-1475. doi:10.1097/ALN.0000000000000176

Zotova, E. G. and Arezzo, J. C. (2013). Non-invasive evaluation of nerve conduction in small diameter fibers in the rat. Physiol $J$ 2013, 254789. doi:10.1155/2013/254789

\section{Conflict of Interest}

JLC and MJM are co-founders of AxoSim, Inc. with equity stakes in the company. LK, HTN, EJ, LM, and ADS are employees of the company.

\section{Acknowledgements}

We would like to acknowledge Ying Xiao from Louisiana State University for her help on histology and transmission electron microscopy imaging. Funding for this research was provided by the NIH (R42TR001270). 